\title{
ADSORCIÓN DE AZUL DE METILENO USANDO UN OXIDO DE GRAFENO PURIFICADO.
}

\author{
Carlos Zamora ${ }^{1}$, Adriana J. Sosa, ${ }^{1}$, Tomas Lozano ${ }^{*}$, Ricardo Garcia ${ }^{1}$, Aarón Melo ${ }^{1}$, Luisiana \\ Morales-Zamudio $^{1}$, Erika Lopez ${ }^{2}$, Sergio Flores ${ }^{2}$, Carlos A. Guerero ${ }^{3}$ Rodolfo Barragan $^{4}$ \\ ${ }^{l}$ Instituto Tecnológico de Ciudad. Madero, Departamento de Ingeniería Química y Bioquímica, Av Primero de Mayo S/N, Col. Los \\ Mangos, C.P. 89440, Cd. Madero, Tamaulipas, México \\ ${ }^{2}$ Centro de Investigación en Materiales Avanzados, Avenida Miguel de Cervantes Saavedra 120, 31136 Chihuahua, Chihuahua \\ ${ }^{3}$ Universidad Autónoma de Nuevo León, Facultad de Ingeniería Mecánica y Eléctrica, Av. Pedro de Alva S/N, 66450 S.N. de los \\ Garza, Nuevo León, México \\ ${ }^{4}$ Facultad de Ingenieria Arturo Narro Siller, Universidad Autonoma de Tamaulipas, Circuito Universitario S/N, Tampico, CP. 89100
}

\section{Resumen}

El azul de metileno se adsorbió sobre la superficie del óxido de grafeno (GO). El óxido de grafeno fue purificado con ácido clorhídrico. El potasio $(\mathrm{K}+)$ y el manganeso $(\mathrm{Mn}+2)$ se eliminaron principalmente durante la purificación. El GO purificado fue llamado GO aniónico de la misma forma el GO no tratado fue llamado GO catiónico, poseedor en su mayor parte de carga positiva. La purificación condujo a una mejor adsorción del azul de metileno. El área específica superficial BET tuvo también una explicación para la adsorción del colorante. El GO aniónico dio un área específica superficial más alta. La adsorción fue determinada por un espectrofotómetro UV-VIS. El contenido de $\mathrm{K}+\mathrm{y}$ $\mathrm{Mn}+2$ fue determinado por Espectroscopía de Dispersión de Energía (EDS). La descomposición del colorante siguió el modelo de Langmuir-Hinshelwood.

Las interacciones entre el colorante catiónico y el GO aniónico fuero clave para la adsorción. Los grupos carbonilo y carboxílico detectados por FTIR en el GO generaron sitios fuertemente negativos

Palabras clave: adsorción, óxido de grafeno, azul de metileno, interacciones, grupos funcionales.

\section{Abstract}

Methylene blue was adsorbed on the surface of graphene oxide (GO). Graphene oxide was purified with hydrochloric acid. Potassium $(\mathrm{K}+)$ and manganese $(\mathrm{Mn}+2)$ were removed mainly during purification. The purified GO was called Anionic GO in the same way the untreated GO was called Cationic GO, which mostly possesses positive charge. Purification led to better adsorption of methylene blue. The specific surface area BET also had an explanation for adsorption of the dye. The anionic GO gave a higher specific surface area. Adsorption was determined by a UV-VIS spectrophotometer. The content of $\mathrm{K}+$ and $\mathrm{Mn}+2$ was determined by Energy Dispersion Spectroscopy (EDS). The decomposition of the dye followed the Langmuir-Hinshelwood model.

The interactions between the cationic dye and the anionic GO were key to adsorption. Carbonyl and carboxylic groups detected by FTIR in the GO generated strongly negative sites.

Keywords: adsorption, graphene oxide, methylene blue, interactions, functional groups.

*Author to whom correspondence should be sent, Phone: +52 (833) 357-4820, e-mail: tomas.lozano@polymtl.ca 


\section{Introducción}

Las corrientes que forman las industrias son las principales causas de las aguas contaminantes debido al uso continuo de diferentes agentes químicos. Los compuestos químicos al mismo tiempo requieren tratamientos específicos dependiendo de la naturaleza de estos productos químicos.

Los contaminantes orgánicos peligrosos producen diferentes efectos adversos para el medio ambiente, así como para la salud pública.

Estos contaminantes se clasifican en tres grupos: Cloruros, Nitroaromáticos y Colorantes.

Los cloruros son los de mayor peligro y son ampliamente utilizados. Sin embargo, estos principales contaminantes están fuertemente regulados debido a que se clasifican como contaminantes prioritarios. Por ahora, el principal está en los compuestos nitroaromáticos y colorantes.

Los colorantes principales son el Rodamine B (RhB), el azul de metileno (MB) y el naranja metilo (MO). Estos colorantes son ampliamente utilizados en las industrias de papel, textil, pinturas, impresión y en la producción de polímeros. Sin embargo, su alta toxicidad y sus efectos potenciales cancerigenos y mutagénicos son señales de advertencias [1].

Los colorantes son compuestos químicos que se aplican a los tejidos (telas) para proporcionar color. La mayoría de los colorantes son moléculas orgánicas complejas que resisten a fuentes como la luz, el agua $\mathrm{y}$ los detergentes.

La descarga de los colorantes en corrientes naturales (efluentes naturales) genera un cambio de color que afecta al paso de la luz solar evitando los procesos fotosíntesis, fotoquímicos y biológicos de la vida acuática.

A nivel mundial, la industria textil utiliza por año más de 10,000 toneladas de colorantes y el $1 \%$ de esta cantidad se descarga sin ningún tratamiento a las corrientes de agua [2].

Los colorantes no se degradan en los procesos tradicionales de tratamientos de agua, de esta manera, algunas estrategias adicionales deben ser utilizadas para su eliminación [3]. Algunos procesos se han aplicado para llevar a cabo la eliminación de colorantes en aguas como: adsorción, coagulación, oxidación avanzada y filtración por membranas.
El método de adsorción de colorantes en soportes sólidos como el carbón activado, es el más eficiente, sin embargo, el alto costo de los carbonos activados comerciales hace de este proceso una alternativa no rentable [4]. Este hecho, conduce a encontrar otros adsorbentes basados en carbono que tienen buenas propiedades de adsorción de compuestos orgánicos.

Entre estos materiales, el óxido de grafeno es un buen candidato para el tratamiento de aguas contaminantes, debido a la disposición de sus átomos (patrones regulares hexagonales) y sus propiedades físicas y químicas. El óxido de grafeno puede actuar como un adsorbente de colorantes en descargas contaminadas [5-7].

En este trabajo de investigación se muestra la eficacia del óxido de grafeno actuando como un componente aniónico para adsorber un colorante catiónico. El óxido de grafeno se comportó bien para el tratamiento de aguas contaminadas con colorantes. $\mathrm{La}$ descomposición del azul de metileno se sugiere ser de primer orden y sigió el modelo de LangmuirHinshelwood.

\section{Parte experimental}

\section{Materiales}

La mayoría de los reactivos como el ácido sulfúrico ( $\mathrm{H} 2 \mathrm{SO} 4,98-99 \%)$, el permanganato de potasio ( $\mathrm{KMnO} 4,99,2 \%$ ), el peróxidode hidrógeno ( $\mathrm{H} 2 \mathrm{O} 2$, $30 \%$ ), y isopropanol, se suministraron por Fermont (México). El azul de etileno fue proporcionado por Eycel México.

\section{Preparación de óxido catiónico y aniónico de grafeno}

GO catiónico GO fue sintetizado a partir de grafito por el método Hummers modificado [8]. En resumen, $5 \mathrm{~g}$ de grafito se añadieron a $130 \mathrm{~mL}$ de $\mathrm{H} 2 \mathrm{O} 4$, estos fueron agitados en un baño de hielo durante 10 minutos. Luego $15 \mathrm{~g}$ de $\mathrm{KMnO} 4$ fue añadido lentamente, y la solución se agitó en un baño de hielo durante 2 horas. La temperatura se elevó y se mantuvo a $35^{\circ} \mathrm{C}$ en un baño de etilenglicol durante 2 horas. Después de eso, se añadieron $230 \mathrm{~mL}$ de agua desionizada, la temperatura de la solución se incrementó a $95^{\circ} \mathrm{C}$ y se agitó durante 15 minutos. Luego, $400 \mathrm{~mL}$ de agua desionizada se añadieron, seguido de $25 \mathrm{ml}$ de $\mathrm{H} 2 \mathrm{O} 2$ (30 Wt\%). La solución fue centrifugada en un Dispositivo Centurion Scientific de la serie K3 (4000 rpm durante 10 minutos) y se lavó hasta que se logró un $\mathrm{pH}$ neutro. La solución de óxido de grafito en agua desionizada fue sometida a 
sonicación con intensidad de $40 \mathrm{kHz}$. Por último, la solución se secó a $70^{\circ} \mathrm{C}$, y el GO catiónico se obtuvo.

El GO aniónico se obtuvo de la misma manera que el GO catiónico. Sin embargo, el GO catiónico se lavó con una solución de $\mathrm{HCl}$ al $5 \%$. Posteriormente fue exfoliado y secado para obtener el GO aniónico. Con esta metodología se eliminaron $\mathrm{K}+\mathrm{y} \mathrm{Mn}+2$ de manera importante [9].

\section{Experimentos de adsorción}

Se preparó la solución de azul de metileno a 50 ppm y luego se añadió posteriormente en un vaso precipitado, 100 gr de cada tipo de óxido de grafeno puesto a la solución. Estos experimentos se llevaron a cabo a los $10,30,60,90,120,150$ y 180 minutos para obtener las isotermas de adsorción. Para cada tiempo de reacción, las soluciones se centrifugaron. El líquido restante se utilizó para obtener espectros UVVIS.

\section{Caracterización}

La superficie específica de ambos óxidos de grafeno se determinó mediante la adsorción de nitrógeno utilizando el modelo Brunauer-Emmett-Teller (BET).

Los patrones de difracción de rayos $\mathrm{X}$ se realizaron en el difractómetro X-PERT (Phillips), utilizando radiación de $\mathrm{Cu} \mathrm{K}(1.54 \AA)$ con $50 \mathrm{kV} \mathrm{y} 40 \mathrm{~mA}$. Las ecuaciones del Bragg y Scherrer se aplicaron para obtener la distancia entre las capas de grafeno (d), la altura media de las capas de apilamiento (h) y el diámetro medio de las capas de apilamiento (D) [10].

Los espectros de infrarrojos de transformada de Fourier (FTIR) se obtuvieron utilizando un espectrofotómetro Nicolet Magna-IR 750. Se prepararon muestras FTIR utilizando $\mathrm{KBr}$ para obtener comprimidos pequeños.

Se utilizó un microscopio electrónico de barrido (JEOL JSM-7610) con un detector de espectroscopia dispersiva de energía (EDS) para medir el porcentaje en peso de potasio y manganeso en los óxidos de grafeno sintetizados. El voltaje de aceleración fue de $10 \mathrm{kV}$.

Los espectros UV-VIS se obtuvieron con un espectrofotómetro Agilent Technologies Cary $60 \mathrm{UV}$ VIS. El líquido restante de los experimentos de adsorción se utilizó para obtener los espectros.

\section{Resultados y discusión}

\section{Área Específica Superficial}

La superficie específica del GO catiónico fue de 96,7 $\mathrm{m} 2 / \mathrm{g}$, mientras que la de GO aniónico fue de 510,62 $\mathrm{m} 2 / \mathrm{g}$.

Caracterización estructural de los materiales grafénicos

La Figura 1 muestra los espectros de transmitancia FTIR de las muestras catiónica y aniónica. El óxido de grafeno muestra los picos característicos relacionados con el carboxilo $(1750 \mathrm{~cm}-1)$, el $\mathrm{C}=\mathrm{C}$ aromático en fenoles (1567 csm-1), los grupos hidroxilo y las mitades de epoxi/éter (1250-1000 cm1) [11]. En esta figura se evidencia la funcionalidad, donde estos grupos dieron el comportamiento aniónico a GO. No se observan diferencias entre el GO catiónico y el GO aniónico.

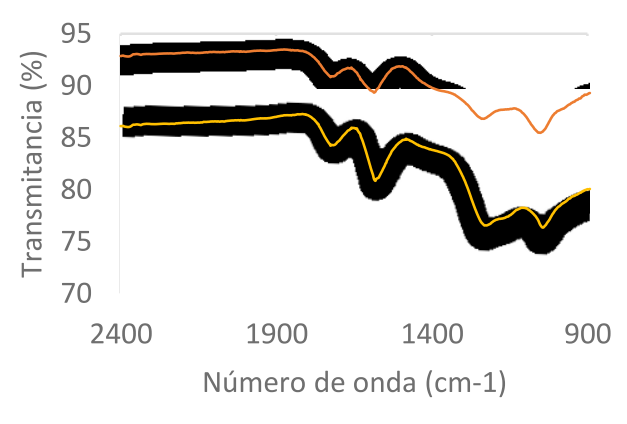

- GO CATIONIC - GO ANIONIC

Figura 1. Espectros de transmitancia FTIR de las muestras GO catiónicas y aniónicas.

La Figura 2 muestra los patrones de difracción del GO catiónico y el GO aniónico. El GO catiónico presenta tres picos a 13,23 y $43^{\circ}$ respectivamente. El GO aniónico presenta principalmente 2 picos para el anglo 2 theta en 12 y 42.5 o respectivamente. El pico característico de la reflexión (002) se observa a 12$13^{\circ}$ en ambos patrones. Este es típicamente el pico principal de ambos GO's. Los picos entre 42.5-43o son impurezas transmitidas desde el precursor del grafito. El GO catiónico muestra un "halo" alrededor de $23^{\circ}$. Este halo está relacionado con la relación carbono amorfo/nanocristalino [12]. 


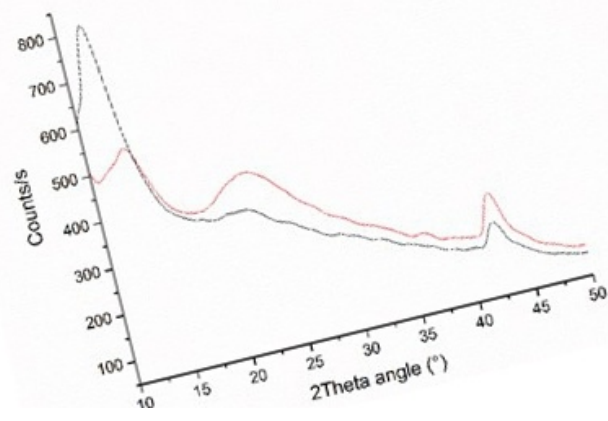

Figura 2. Patrones de difracción de ambos óxidos de grafeno

La distancia entre las capas de grafeno (d), la altura media de las capas de apilamiento (h) y el FWHM se presentan en la Tabla 1. El óxido de grafeno catiónico consta de 2 capas de grafeno, un valor de distancia entre capas $\mathrm{d}=0.67 \mathrm{~nm}$. El óxido de grafeno aniónico presenta 4 capas de grafeno con una distancia entre capas $\mathrm{d}=0.72 \mathrm{~nm}$.

Tabla 1. Parámetros de GO catiónico y GO aniónico resultantes de los patrones XRD.

\begin{tabular}{|c|c|c|c|c|c|}
\hline \multirow{2}{*}{ Muestra } & $\begin{array}{c}2 \theta \\
\left({ }^{\circ}\right)\end{array}$ & $\begin{array}{c}\mathrm{D} \\
(\mathrm{nm})\end{array}$ & $\begin{array}{c}\text { FWHM } \\
\left(^{\circ}\right)\end{array}$ & $\begin{array}{c}\mathrm{H} \\
(\mathrm{nm})\end{array}$ & $\mathrm{N}$ \\
\hline Ir & 13 & 0.67 & 5.87 & 1.49 & 2 \\
\hline Rgo & 12.24 & 0.72 & 2.85 & 2.93 & 4 \\
\hline
\end{tabular}

Notación. $d$ : distancia media entre capas; FWHM: ancho a la mitad del máximo; $h$ : altura media de las nanocapas apiladas, $\mathrm{n}$ : número promedio de nanocapas apiladas.

La espectroscopia dispersiva de energía se llevó a cabo en ambos óxidos de grafeno para medir el porcentaje en peso de potasio y manganeso, esto se hizo para corroborar la eliminación de estas impurezas. La Figura 3a muestra el \% de potasio y manganeso del GO catiónico, mientras que la Figura $3 \mathrm{~b}$ muestra el \% de potasio y manganeso para el GO aniónico. Hubo una clara disminución en $\mathrm{K}+\mathrm{y} \mathrm{Mn}+2$ después de la adición de $\mathrm{HCl}$ al GO catiónico para eliminar estas impurezas. El \% de $\mathrm{K}+$ se redujo de 0.2 a 0.1 , mientras que para $\mathrm{Mn}+2$ el $\%$ en peso se redujo de 0.5 a 0.2 . La eliminación de $\mathrm{K}+\mathrm{y} \mathrm{Mn}+2$ condujo a un comportamiento más aniónico del GO.

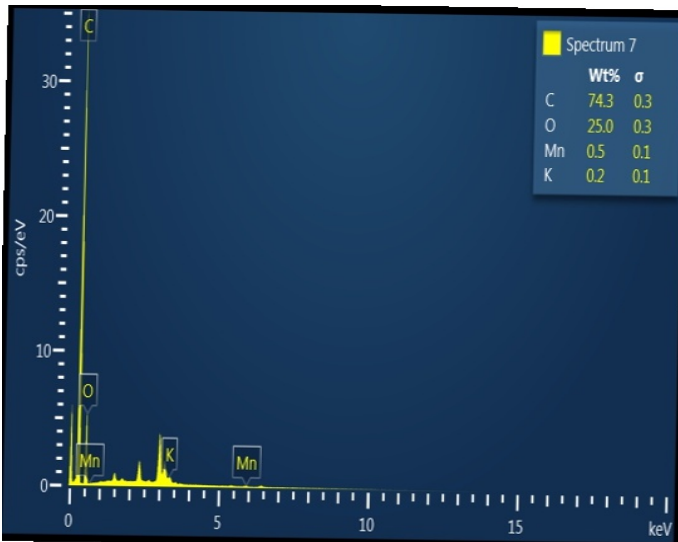

Figura 3a. Espectro EDS del óxido grafeno catiónico.



Figura 3b. Espectro EDS del óxido de grafeno aniónico.

\section{$\underline{\text { Resultados de adsorción }}$}

En las figuras $4 \mathrm{a}$ y $4 \mathrm{~b}$ respectivamente muestran los espectros UV-VIS de las muestras del azul de metileno decolorado con óxido de grafeno. El colorante tiene una adsorción máxima a $665 \mathrm{~nm}$. Esta longitud de onda se utilizó como referencia para determinar la capacidad de adsorción del GO. A partir de estas figuras se puede ver que el óxido de grafeno presenta una capacidad de eliminación del azul de metileno. La intensidad del pico de azul de metileno se reduce con la presencia de GO, con el tiempo de reacción. La mejor adsorción se obtuvo con GO aniónico. La adsorción es favorecida con la presencia de grupos funcionales en el GO para atrapar partículas de colorante. Estos resultados sugieren una afinidad del GO con el azul de metileno catiónico. 




Figura 4a. Espectros UV-VIS de experimentos con GO catiónico.

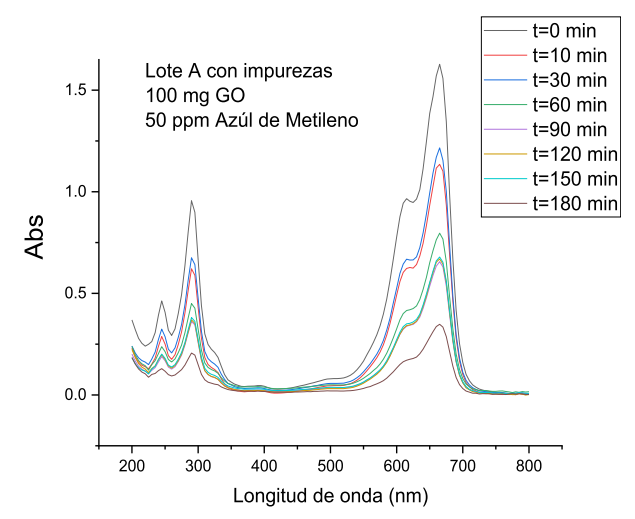

Figura 4b. Espectros UV-VIS de experimentos con GO aniónico.

El azul de metileno es una molécula catiónica que puede generar interacciones con los grupos funcionales del GO.

Los grupos carboxílico y carbonilo generan sitios muy negativos que inducen altas fuerzas electrostáticas para las atracciones e interacciones con partículas catiónicas $[13,14]$.

La capacidad catiónica teórica del azul de metileno, así como la capacidad aniónica del óxido de grafeno, sugieren que las fuerzas de atracción son las responsables de la adsorción del azul de metileno en la superficie del GO.

Los resultados muestran que GO con menos cationes de $\mathrm{K}+1$ y $\mathrm{Mn}+2$ (GO aniónico) dio mejores resultados que el que tiene más contenido de estos cationes (GO catiónico). Esto es claro que GO con menos cationes hace una molécula con un carácter más aniónico, que puede tener más interacciones con el colorante catiónico.
Por cierto, la eliminación de $\mathrm{K}+\mathrm{y} \mathrm{Mn}+2$ en $\mathrm{GO}$ también aumentó el área específica superficial. Los sitios libres (sin cationes) en el óxido de grafeno son muy importantes para llevar a cabo una mejor adsorción.

La Figura 5 muestra el comportamiento de la concentración de azul de metileno sin GO, con GO catiónico y GO aniónico contra el tiempo. Se puede ver que el azul de metileno no presenta ninguna decoloración en este lapso. El azul de metileno se degrada más rápido con el GO aniónico que con el GO catiónico. La descomposición se debe al comportamiento aniónico del GO, así como, su mayor superficie específica aumentando la cantidad de moléculas adsorbidas del colorante en la superficie del GO.



O WITHOUT GO O CATIONIC GO O ANIONIC GO

Figura 5. Evolución de la concentración del azul de metileno (50ppm) durante su descomposición con GO.

En la Figura 6 se muestra el comportamiento - $\ln \left(C / C_{0}\right)$ contra tiempo del sistema azul de metileno/GO. Se puede ver un comportamiento lineal. Esto sugiere que la reacción de descomposición es de primer orden y sigue el modelo LangmuirHinshelwood. La pendiente de cada línea da la constante la velocidad de reacción $(k)$. El tiempo de vida media $\left(t_{1 / 2}\right)$, para las reacciones de primer orden, se puede calcular con la siguiente ecuación [15]:

$$
t_{1 / 2}=\frac{\ln 2}{k}
$$

En la tabla 2 se dan las constantes de velocidad de reacción $(k)$ y tiempo de vida media $\left(t_{1 / 2}\right)$ para cada reacción llevada a cabo. 


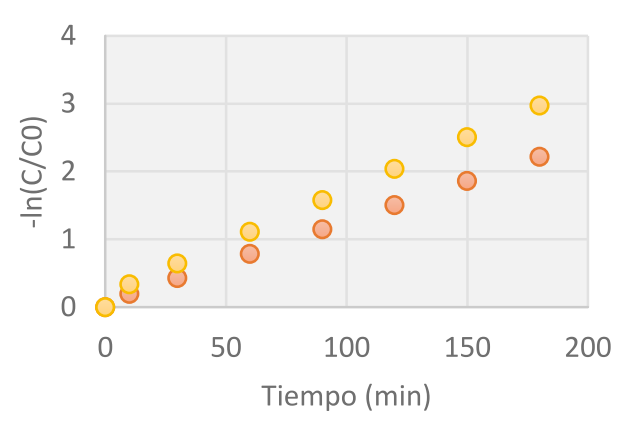

OCATIONICGO OANIONIC GO

Figura 6. Gráfica del $-\ln (\mathrm{C} / \mathrm{CO})$ contra el comportamiento del azul de metileno con GO en el tiempo.

Tabla 2. Velocidad de reacción constante $(k)$ y vida media $\left(t_{1 / 2}\right)$ del modelo Langmuir-Hinshelwood.

\begin{tabular}{|c|c|c|}
\hline & $\left.\mathbf{k} \times \mathbf{1 0}^{\mathbf{3}} \mathbf{( m i n}^{-\mathbf{1}}\right)$ & $\mathbf{t}_{\mathbf{1 / 2}}(\mathbf{m i n})$ \\
\hline GO Catiónico & 0.0119 & 58.25 \\
\hline GO Aniónico & 0.0138 & 50.23 \\
\hline
\end{tabular}

Aquí se muestra que el óxido de grafeno aniónico aceleró la descomposición y dio un menor tiempo para degradar las moléculas de azul de metileno.

\section{Conclusiones}

Una vez llevada a cabo la investigación y experimentación se pueden concluir diversas hipótesis. El azul de metileno se adsorbió sobre la superficie del óxido de grafeno (GO). El óxido de grafeno fue purificado con ácido clorhídrico. $\mathrm{K}+\mathrm{y}$ $\mathrm{Mn}+2$ se eliminaron principalmente durante la purificación. La purificación llevó a una mejor adsorción del azul de metileno. El área específica superficial también tuvo una explicación para la adsorción del colorante. El GO aniónico dio un área específica superficial más alta.

Los grupos carboxílico y carbonílico generan sitios muy negativos en GO lo que llevó a altas fuerzas electrostáticas para las atracciones e interacciones con el azul de metileno catiónico.

Los resultados obtenidos sugieren que las fuerzas de atracción son las responsables de la adsorción del azul de metileno en la superficie del GO.

Se sugiere que la reacción de descomposición del azul de metileno es de primer orden y sigue el modelo Langmuir-Hinshelwood. El óxido de grafeno aniónico aceleró la descomposición y dio un menor tiempo para degradar las moléculas de azul de metileno.

Debido a los resultados obtenidos en este trabajo de investigación, se puede proponer que el óxido de grafeno purificado es una muy buena alternativa para el tratamiento de aguas contaminadas con colorantes.

\section{Referencias}

[1] Cui, X., Zheng, Y., Tian, M., \& Dong, Z. 2017. Palladium nanoparticles supported on SiO2@Fe3O4@m$\mathrm{MnO} 2$ mesoporous microspheres as a highly efficient and recyclable catalyst for hydrodechlorination of 2,4 dichlorophenol and reduction of nitroaromatic compounds and organic dyes. Molecular Catalysis, 433, 202-211.

[2] Yagub, M., Sen, T., Ang, H. 2012. Equilibrium, kinetics, and thermodynamics of methylene blue adsorption by pine tree leaves. Water Air Soil Pollut, 223(8), 5267-82.

[3] Robinson, T., Mcmullan, G., Marchant, R., \& Nigam, P. 2001. Remediation of dyes in textile effuent: a critical review on current treatment technologies with a proposed alternative, Bioresource technology, 77, 247-255.

[4] FORGACS, Esther, CSERHÁTI, Tibor, OROS, Gyula, "Removal of synthetic dye from wastewaters: a rewiew", Environment International 30 953-971, 2004

[5] YANG, Sheng-Tao, et al, "Removal of methylene blue from aqueous solution by graphene oxide", Journal of Colloid and Interfase Science, 359, 24-29, 2011.

[6] ZHANG, Wenjie, et al, "Fast and Cosiderable Adsorption of Methylene Blue Dye onto Graphene Oxide", Bull Environ Contam Toxicol 87:86-90, 2011.

[7] RAMESHA, G.K. et al, "Graphene and graphene oxide as effective adsorbents toward anionic and cationic dyes", Journal of Colloid and Interface Science. 361 270-277, 2011

[8] Z. Dai, K. Wang, L. Li, T. Zhang, Synthesis of NitrogenDoped Graphene with Microwave, International Journal of Electrochemical Science 8 (2013) 9384-9389

[9] N. G. Barbakadze, V. G. Tsitsishvili, T. V. Korkia, Z. G. Amiridze, N. V. Jalabadze and R.V. Chedia, " SYNTHESIS OF GRAPHENE OXIDE AND REDUCED GRAPHENE OXIDE FROM INDUSTRIAL GRAPHITE FOIL WASTES”, Eur. Chem. Bull. 2018, 7(11), 329-333

[10] B.E. Warren, Phys. Rev. 59 (1941) 693-698

[11] Pavia, D. L. Lampman, G. M. Kriz. G. S. (s.f.). Introduction to spectroscopy, Third Edition. 14, 15, 463.

[12] Guerrero-Contreras Jesus, Caballero-Briones F, Materials Chem Phys (2015) 153: 209-220. 
[13] Liu, X., Huang, Y., Duan, S., Wang, Y., Li, J., Chen, Y., Wang, X. 2016. Graphene oxides with different oxidation degrees for Co (II) ion pollution management. Chemical Engineering Journal, 302, 763-772.

[14] Dreyer, D. R., Park, S., Bielawski, W., \& Ruoff, R. S. 2010. The chemistry of graphene oxide. Chem. Rev. 39228 240.

[15] Levenspiel O, Chemical Reaction Engineering, 3rd Edition, 1999, John Wiley and Sons. 